\title{
The role of the Pharmaceuticals and Medical Devices Agency and healthcare professionals in post-marketing safety
}

\author{
Kazuhiko Mori • Meguru Watanabe • \\ Naoya Horiuchi $\cdot$ Atsushi Tamura \\ Hiromu Kutsumi
}

Received: 26 February 2014/ Accepted: 3 March 2014/Published online: 22 March 2014

(C) Springer Japan 2014

\begin{abstract}
The development of drugs and medical devices is necessary for medical progress; however, safety measures need to be put in place to protect the health of the population. In order to ensure the safety of drugs and medical devices, it is important to determine measures for appropriate management of risks at any time during the development phase, the regulatory review and the post-marketing phase. Adverse events detected in clinical trials are limited due to the restricted numbers of patients enrolled in the trials. Therefore, it is almost impossible to predict rare serious adverse events during the post-marketing phase. The revised Pharmaceutical Affairs Act was established in Japan in November 20, 2013. The new act focuses on increased safety of drugs and medical devices. The Pharmaceuticals and Medical Devices Agency (PMDA) is the regulatory authority in Japan that promotes safety measures from the development phase through to the post-marketing
\end{abstract}

\section{K. Mori}

Foundation for Biomedical Research and Innovation, 2-2

Minatojima Minamimachi, Chuo-ku, Kobe 650-0047, Japan

M. Watanabe $(\square)$

Pharmaceuticals and Medical Devices Agency, Shin-

Kasumigaseki Building, 3-3-2 Kasumigaseki, Chiyoda-ku,

Tokyo 100-0013, Japan

e-mail: watanabe-meguru@pmda.go.jp

\section{N. Horiuchi · A. Tamura}

Kansai Branch, Pharmaceuticals and Medical Devices Agency, Knowlege Capital Tower C-9F, 3-1 Ofuka-cho, Kita-ku,

Osaka 530-0011, Japan

\section{H. Kutsumi}

Department of Gastroenterology, Kobe University, Graduate

School of Medicine, 7-5-1 Kusunoki-cho, Chuo-ku,

Kobe 650-0017, Japan

e-mail: hkutsumi@med.kobe-u.ac.jp phase. In the post-marketing phase, the PMDA collects information from the medical product companies and healthcare professionals, as well as instructing and advising them with regard to post-marketing safety measures for each drug and medical device. Since Japan has a national health insurance system, a new drug or a medical device is available throughout the country when the drug price or medical fee is listed in the National Health Insurance price list. Healthcare professionals in medical institutions must learn about the drugs and medical devices they handle, and should make an effort to maintain patient safety. The PMDA medi-navi is a very useful electronic mail delivery service that provides critical information for protecting patients from health hazards caused by adverse events. The 'risk management plan' is also important as it contains important information about safety profile and post-marketing measures of a new drug.

Keywords Post-marketing safety measures . Regulatory authority $\cdot$ PMDA

\section{Introduction}

The development of drugs and medical devices is necessary for medical progress; however, safety measures need to be put in place to protect the health of the population. Postmarketing regulation and pre-marketing regulations are both very important with regard to safety measures. The Pharmaceuticals and Medical Devices Agency (PMDA) is the regulatory authority in Japan. In cooperation with the PMDA, the drug/device developer undertakes most of the pre-marketing safety measures; however, the healthcare professionals including clinicians working at medical institutions play an important role in the post-marketing 
safety measures. As people who are working or have worked at the PMDA and have the understanding and cooperation of many clinicians in the national health system, we describe the post-marketing safety measures in Japan.

\section{Post-marketing safety measures: the role of the PMDA and the PMDA clinical reviewers}

Ten years after the PMDA was established in April 2004, approximately 50 medical doctors work for the PMDA among 700 full-time employees. Most of them have many years clinical training in various medical specialties with board certification in each specialty. With expertise and experience in their own fields, they work in the approval, review, and consultation processes at the PMDA. The PMDA clinical reviewers play a crucial role in post-marketing phase vigilance and safety measures in pharmaceuticals and medical devices.

PMDA clinical reviewers at the Office of Safety mainly work on reinforcement of post-marketing safety measures. Their tasks include reading and understanding adverse drug reactions (ADRs) reported daily to the PMDA, and evaluate the necessity to take action for reported adverse reactions. The number of reports regarding ADRs has been increasing annually, partly because the PMDA has been dealing with the issue of so-called 'drug lag' to approve increased numbers of the drugs each year (Fig. 1).

PMDA clinical reviewers at the Office of Safety collaborate with other PMDA clinical reviewers who give advice on safety reports in their own specialty. In addition to collaboration with their colleagues, PMDA clinical reviewers at the Office of Safety play a pivotal role in liaisons between the PMDA and healthcare professionals at medical institutions.

Healthcare professionals in medical institutions should fully understand possible ADRs before administering a drug to a patient. They can obtain information on new drugs or medical devices on the PMDA information homepage [1, 2], the PMDA website [3] and the homepage

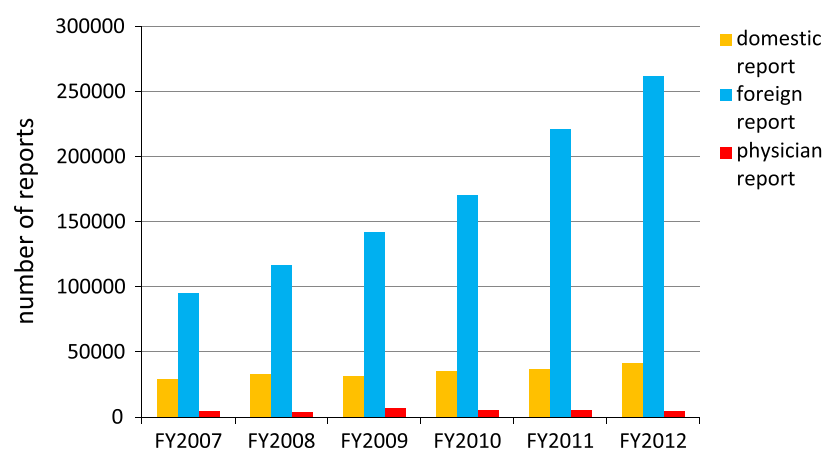

Fig. 1 Annual numbers of ADR case reports of respective companies. The information is accumulated in a clinical trial called 'Chiken' in Japan. However, ADRs detected during the clinical trial are limited due to the restricted number of patients enrolled in the trial. Several thousand patients are usually enrolled in a clinical trial for a new drug. Therefore, it is almost impossible for healthcare professionals to predict or suspect a rare serious ADR, i.e., one in 10,000 cases, in the early post-marketing phase.

\section{The revised Pharmaceutical Affairs Act and reinforcement of safety issues}

The revised Pharmaceutical Affairs Act was established on November 20, 2013, and came into force on November 27, 2013 [4]. The new act is called the 'Law about reservation of quality, efficacy, and safety, such as drugs and medical devices' ('Pharmaceuticals and Medical Devices Law' in short). The new act focuses on increased safety of drugs, medical devices and tissue-engineered medical products.

Before revision, Article 77 of the Pharmaceutical Affairs Act focused on the safety of drugs and medical devices. Article 77 describes offering information, educating for an appropriate usage, preventing hazards, reporting adverse events and non-conformance, all of which are described in Article 68 in the revised law.

The articles in the revised law say that medical product companies, and healthcare professionals including dentists and pharmacists are responsible for patient safety. The following three points are of note [5].

1. The revised law indicates necessary regulatory actions to prevent hazards of healthcare and hygiene issues.

2. Related healthcare professionals are responsible for guaranteeing efficacy and safety of pharmaceuticals and medical devices.

3. Marketing Authorization Holders (MAHs) of pharmaceuticals and medical devices must provide package inserts based on updated data, and submit them to the Ministry of Health, Labor, and Welfare (MHLW).

It is essential that clinicians are aware of the above information. In particular, the second point is described in detail in Act no. 5, the text of law 1 [6] as follows:

[Physicians, dentists, pharmacists, veterinarians and all other healthcare professionals should deepen their understanding of safety and efficacy of pharmaceuticals, etc., and make an utmost effort to deliver precise and proper information to those who are to receive those pharmaceuticals, etc. and those who purchase and receive them.]

In other words, as healthcare professionals, clinicians must learn about the drugs and medical devices they handle, and explain them to patients and their families in understandable language. 
Act no. 4, the text of law 1 refers to the responsibility of any individual or institute involved in the business of pharmaceuticals and medical devices.

[Founders of hospitals and clinics must share any information among those who it may concern, and maintain quality, efficacy and safety of certain pharmaceuticals, etc. and prevent the occurrence and expansion of health hazards.]

Not only clinicians, but hospitals, clinics, pharmaceutical companies, and pharmacies, as a juristic person, should make an effort to maintain patient safety.

\section{Characteristics of post-marketing safety measures in Japan and the role of the PMDA in management}

Among the post-marketing safety measures in Japan, the PMDA mainly focuses on evaluating ADRs reported via early post-marketing phase vigilance (EPPV). In the development phase of a new drug, the safety and efficacy are evaluated based on updated information they receive. However, it is difficult to identify all possible adverse events in this phase due to the limited number of the patients enrolled in the clinical trials in a restricted period of time.

On the other hand, in the early post-marketing phase, the drug is given to more patients than in the clinical trials. A greater number of patients will provide a variety of different medical backgrounds which can lead to early detection of ADRs that are difficult to identify in the development phase. Such adverse events have often been observed within 6 months of the drug being put on the market. Since Japan has a national health insurance system, a new drug is available throughout the country when the drug prices and reimbursements are listed in the National Health Insurance drug price list. In Japan, these processes usually take 60-90 days from approval.

For drugs approved after October 2001, the Pharmaceutical Affairs Act requires pharmaceutical companies to make an effort to detect ADRs in the early EPPV and, if necessary, to take appropriate safety measures for the adverse events. Employees from the pharmaceutical company have to visit health professionals who use the new drug at least once every 2 weeks during the first 2 months after the drug is put on the market. They have to provide health professionals with information on the proper use of the drug, and collect data on any adverse reactions. If any ADRs are identified, they have to be reported to the PMDA immediately [7].

After the first 6 months, the pharmaceutical company is responsible for reporting any adverse reactions to the PMDA, including infections from biological products as long as the drug is on the market. In cases of serious
ADRs, the MHLW requires that package inserts for the drug should be revised based on the analysis by the PMDA. All the collected data on ADRs are reviewed and evaluated on the basis of risk-benefit balance when the drug is being re-evaluated for approval. A new drug is reevaluated 4-10 years after approval, while a drug containing a new compound is re-evaluated within 8 years (Fig. 2).

The PMDA is responsible for obtaining safety information on a new drug from the pharmaceutical company, and delivering the information to all healthcare professionals and pharmacies that are handling the new drug. As mentioned above, during the period when employees from the pharmaceutical company are in frequent contact with healthcare professionals using the new drug, it is possible to know whether any ADRs have been identified and appropriate safety measures have been taken. When necessary, the MHLW requires the pharmaceutical company to issue an urgent safety alert (a 'blue letter') or an emergency safety alert ('yellow letter').

The PMDA promotes the system of post-marketing safety measures, including collecting information from pharmaceutical companies and healthcare professionals, as well as instructing and advising the pharmaceutical companies with regard to post-marketing safety measures for each drug. Clinicians who learn the system can benefit from the information about new drugs provided by the pharmaceutical companies.

The PMDA clinical reviewers at the Office of Safety give advice to the pharmaceutical companies based on their expertise and experience in the 'real world' medical setting so that appropriate safety measures can be taken at the medical institutions.

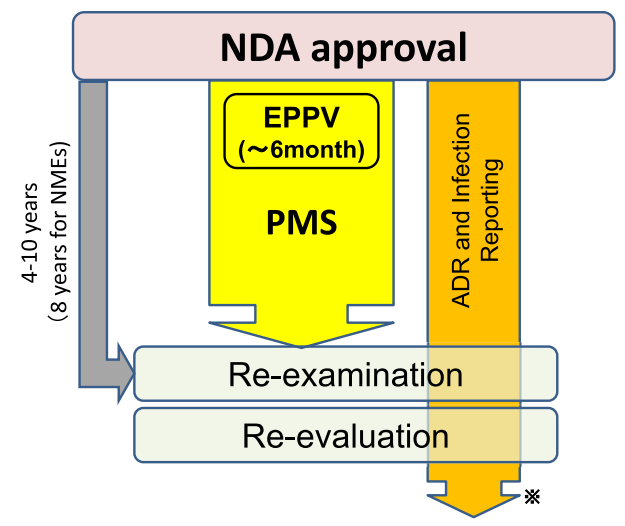

Fig. 2 The current framework for post-marketing safety measures. $N D A$ new drug application, $E P P V$ early post-marketing phase vigilance, $A D R$ adverse drug reactions, $N M E$ new molecular entity, $※$ : ADR and infection reporting are continued 
Fig. 3 Concept of the Japanese RMP. RMP Risk Management Plan, $P v P$ Pharmacovigilance Plan, RiskMAP Risk

Minimization Action Plan, PharmacoEpi Study PharmacoEpidemiology Study, EPPV early post-marketing phase vigilance, $*$ : burden on health care providers should be taken into consideration

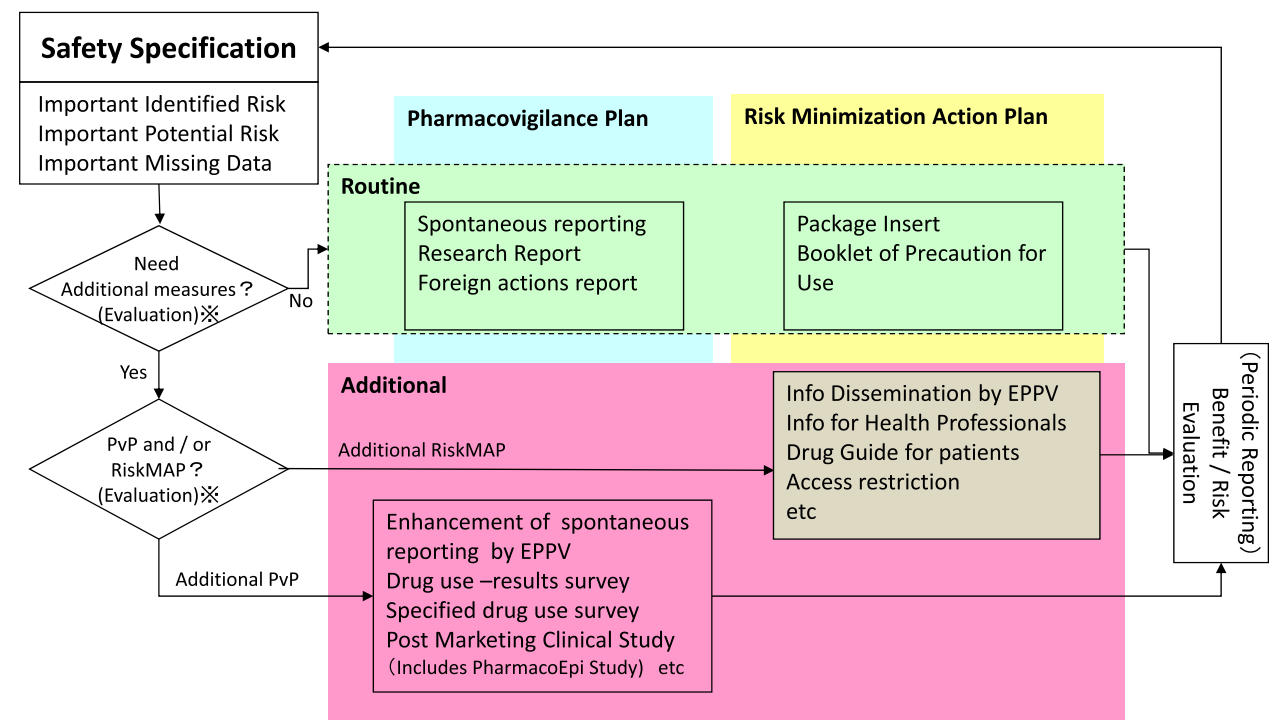

New systems in post-marketing safety measures: PMDA medi-navi and Risk Management Plan

PMDA promotes post-marketing safety measures using two new systems. PMDA medi-navi is an electronic mail delivery service for registered users. At the end of 2013, there were approximately 100,000 users registered to use PMDA medi-navi. Significant safety information about drugs must be updated and delivered directly to healthcare professionals in a timely fashion. In this regard, e-mail is also an effective system [8].

As mentioned above, in addition to the ADRs reported during the clinical trials, other adverse events can be identified during EPPV and reported to the PMDA. The PMDA evaluates reports and takes prompt action to provide necessary information to healthcare professionals. The PMDA medi-navi is an extremely useful system for this purpose, providing critical information for preventing patients from health hazards from ADRs.

Another new post-marketing safety measure which healthcare professionals need to know about is the 'Risk Management Plan' (RMP). The RMP comprises two partsthe pharmacovigilance plan to identify possible ADRs as early as possible and the risk minimization action plan to minimize health hazards by known ADRs. After April 2013, pharmaceutical companies have to provide an RMP after discussing safety of the new drug with the PMDA throughout the review process for the new drug, and provide necessary information in a precise and concise fashion to the PMDA, whose website displays this information. The RMP contains important information about the safety profile and postmarketing measures for a new drug [9-11].

A major concept of the RMP is based on the International Conference on Harmonization of Technical
Requirements for Registration of Pharmaceuticals for Human Use guideline (ICH-E2E) [12]. In order to ensure the safety of drugs, it is important to determine measures for appropriate management of the risks associated with the drugs at any time during the development phase, the regulatory review and the postmarketing phase. Therefore, it is important to document such current actions for each drug and to share the information among the relevant personnel to ensure further improvements of post-marketing safety measures [13, 14] (Fig. 3).

Each RMP appearing on the PMDA website provides information about the pharmacovigilance plan and risk minimization action plan to the healthcare professionals who use the new drug. The PMDA clinical reviewers at the Office of Safety collaborate with pharmaceutical companies to create a precise and concise RMP.

\section{Summary}

We have described the role of the PMDA and the PMDA clinical reviewers in post-marketing safety measures. The PMDA clinical reviewers contribute to promoting the reinforcement of post-marketing safety measures and providing precise and concise RMPs, based on reports from the 'real world' medical setting.

\section{Conclusion}

We hope that we have helped to provide a better understand of post-marketing safety measures for pharmaceuticals and medical devices, as well as to understand the role 
of the PMDA, the PMDA reviewers and healthcare professionals in post-marketing safety measures.

\section{Disclosure}

Conflict of Interest: Kazuhiko Mori, Meguru Watanabe, Naoya Horiuchi, Atsushi Tamura and Hiromu Kutsumi declare that they have no conflict of interest.

This review article does not have relevant issues of human/animal rights and informed consent disclosure to be reported.

\section{References}

1. Iyakuhin Kanren Jouhou. Pharmaceuticals and Medical Devices Agency. http://www.info.pmda.go.jp/info/syounin_index.html. Accessed 20 February 2014 (In Japanese).

2. Iryoukiki Kanren Jouhou. Pharmaceuticals and Medical Devices Agency. http://www.info.pmda.go.jp/info/syounin_kiki_index. html. Accessed 20 February 2014 (In Japanese).

3. Shinyaku no Kaihatsu, Shinsa, Shihan go Anzentaisaku no Tsunagari ni tsuite. Pharmaceuticals and Medical Devices Agency. http://www.pmda.go.jp/guide/kagakuiinkai/iyaku/h241101gijishi dai/file/shiryo2-1.pdf. Accessed 20 February 2014 (In Japanese).

4. Yakuji-hou (Pharmaceutical Affairs Act). http://law.e-gov.go.jp/ htmldata/S35/S35HO145.html\#100000000001000000000000000 0000000000000000000000000000000000000000000000000000. Accessed 20 February 2014 (In Japanese).

5. Yakuji-hou no ichibu wo Kaitei suru Houritsu no Gaiyou. Pharmaceuticals and Medical Devices Agency. http://www.pmda.go. jp/guide/hyougikai/25/h251226shinsaanzen2/file/sanko3.pdf. Accessed 20 February 2014 (In Japanese).
6. Yakuji-hou (Pharmaceutical Affairs Act). http://law.e-gov.go.jp/ cgi-bin/idxmiseko.cgi?H_RYAKU $=\% 8 \mathrm{f} \%$ ba $\% 8 \mathrm{e} \% 4 \mathrm{f} \% 8 \mathrm{c} \% \mathrm{dc} \%$ $96 \% 40 \% 88 \%$ ea $\% 8 \mathrm{e} \% 6 \mathrm{c} \% 8 \mathrm{c} \%$ dc\&H_NO=\%95\%bd\%90\%ac\% 93\%f $1 \% 8 \mathrm{f} \% 5 \mathrm{c} \% 8 \mathrm{c} \% \mathrm{dc} \% 94 \% 4 \mathrm{e} \% 8 \mathrm{f} \% 5 \mathrm{c} \% 88 \%$ ea $\% 8 \mathrm{c} \% 8 \mathrm{e} \% 93 \%$ f1\%8f\%5c\%8e\%b5\%93\%fa\%96\%40\%97\%a5\%91\%e6\%94\%aa\% $8 \mathrm{f} \% 5 \mathrm{c} \% 8 \mathrm{e} \% 6 \mathrm{c} \% 8 \mathrm{~d} \% 86 \& \mathrm{H} \_\mathrm{PATH}=/$ miseko/S35HO145/H25HO 084.html. Accessed 20 February 2014 (In Japanese).

7. Shihan Chokugo Chousa ni kansuru Jouhou. Pharmaceuticals and Medical Devices Agency. http://www.info.pmda.go.jp/shinyaku/ shihan_index.html. Accessed 20 February 2014 (In Japanese).

8. Iyakuhin Iryoukiki Jouhou Haishin Service. Pharmaceuticals and Medical Devices Agency. http://www.info.pmda.go.jp/info/idxpush.html. Accessed 20 February 2014 (In Japanese).

9. Iyakuhin Risk Kanri Keikaku Shishin. Pharmaceuticals and Medical Devices Agency. http://www.info.pmda.go.jp/iyaku/file/ h240411-001.pdf. Accessed 20 February 2014 (In Japanese).

10. Iyakuhin Risk Kanri Keikaku no Satei ni tsuite. Pharmaceuticals and Medical Devices Agency. http://www.info.pmda.go.jp/iyaku/ file/h240426-001.pdf. Accessed 20 February 2014 (In Japanese).

11. Iyakuhin Risk Kanri Keikakusho no Kouhyou ni tsuite. Pharmaceuticals and Medical Devices Agency. http://www.info. pmda.go.jp/iyaku/file/h250304-001.pdf. Accessed 20 February 2014 (In Japanese).

12. ICH Harmonised Tripartite Guideline: Pharmacovigilance Planning. Pharmaceuticals and Medical Devices Agency. http://www. pmda.go.jp/ich/e/e2e_05_9_16e.pdf. Accessed 20 February 2014.

13. RMP:Risk Management Plan. Pharmaceuticals and Medical Devices Agency. http://www.info.pmda.go.jp/rmp/rmp_index. html. Accessed 20 February 2014 (In Japanese).

14. Risk Management Plan (RMP). Pharmaceuticals and Medical Devices Agency. http://www.pmda.go.jp/english/service/rmp. html. Accessed 20 February 2014. 